\title{
Importance Ethnobotanique Et Des Menaces Sur Le Délice Du Boboye, Neocarya Macrophylla (Sabine) Prance, (Chrysobalanaceae) Dans La Vallée Fossile Du Dallol Bosso (Niger)
}

\author{
Aboubacar Kolafane, \\ Biotechnologiste, Département de Biologie, Faculté des Sciences et \\ Techniques, Université Abdou Moumouni de Niamey, Niger
}

Douma Soumana,

Ecologie forestière, Département de Biologie, Faculté des Sciences et

Techniques, Université Abdou Moumouni de Niamey, Niger

\section{Adamou Mahaman Moustapha,}

Département des Sciences Fondamentales, Faculté d'Agronomie,

Université Abdou Moumouni de Niamey, Niger

Hama Insa Abdoulaye,

Département des Sciences Fondamentales, Faculté d'Agronomie,

Université Abdou Moumouni de Niamey, Niger

\section{Agundez Dolores,}

National Institute for Agriculture and Food Research and Technology (INIA), Forest Research Centre (CIFOR). Ctra de La Coruña, Madrid, Spain; iuFOR, Sustainable Forest Management Research Institute, University of Valladolid \& INIA

\section{Sidikou Djermakoye Seyni Ramatou,}

Biologiste, Physiologiste et Biotechnologiste, Département de Biologie,

Faculté des Sciences et Techniques, Université Abdou Moumouni de Niamey, Niger

Doi: 10.19044/esj.2018.v14n12p160 URL:http://dx.doi.org/10.19044/esj.2018.v14n12p160

\begin{abstract}
The present study conducted in the Dallol Bosso fossil valley aims to know the local perception of the level of threat on the Neocarya macrophylla species in order to provide indicators of sustainable management of the natural stand. To do this, surveys were conducted in two agro-ecological zones.The collection of information was carried out through a simple questionnaire based on the woody stand, the sources of supply and the part of the plant taken from the woody food species, as well as the threats they suffer and their causes. It appears that the most dominant species is Neocarya macrophylla; a
\end{abstract}


multipurpose species among others, used for food, medicine and wood.The survey also reveals the presence of twenty four (24) ligneous foods species among which eighteen (18) are common to both zones. Analyses showed, the excessive cutting of the wood and the unfavorable climatic conditions as the main factors resulting on potential threat of these species. At the same time, threats on Neocarya macrophylla, a characteristic species of Dallol Bosso valley were identified on the field.

Keywords: Non-wood forest products, ethnobotanical survey, food security, Neocarya macrophylla, Dallol Bosso

\section{Résumé}

La présente étude, conduite dans la vallée fossile du Dallol Bosso, vise à connaitre la perception locale du niveau de menace sur l'espèce Neocarya macrophylla en vue de fournir des indicateurs de gestion durable $d u$ peuplement naturel. Pour ce faire, des enquêtes ont été menées dans deux zones agroécologiques. La collecte des informations a été effectuée à travers un questionnaire simple articulé autour du peuplement ligneux, les sources d'approvisionnement et les organes prélevés des espèces ligneuses alimentaires ainsi que les menaces qu'elles subissent et leurs causes. Il ressort que l'espèce la plus dominante est Neocarya macrophylla. Cette espèce est à usages multiples dont entre autres l'usage alimentaire, l'usage médicinal et l'usage énergétique. L'enquête révèle également la présence de vingt- quatre (24) espèces ligneuses alimentaires dont dix- huit (18) sont communes aux deux zones. Ainsi, à l'issue des analyses, la coupe abusive du bois et les conditions climatiques défavorables constituent la potentielle menace sur ces espèces. Parallèlement, des constats de menaces sur Neocarya macrophylla, espèce caractéristique du Dallol Bosso ont été particulièrement énumérés sur le terrain.

Mots-clés : Produits forestiers non ligneux, enquête ethnobotanique, sécurité alimentaire, Neocarya macrophylla, Dallol Bosso

\section{Introduction}

Le Niger a connu plusieurs événements extrêmes liés aux variabilités climatiques actuelles et passées durant ces dernières décennies. Les plus importants sont les sécheresses de 1972/73 et 1984/85 et la dégradation des terres (Douma, 2009). Cette situation a fortement fragilisé les systèmes de production fondés sur les cultures pluviales. On assiste dès lors à l'effondrement des productions agricoles consécutif à une baisse sensible de la fertilité des sols (Akpo et al., 2003 ; Habou et al., 2015). Pour combler le déficit, les producteurs ont recours à l'augmentation des superficies emblavées 
soit par la déforestation, soit par une mise en valeur des terres marginales. Le recours à de nouvelles terres s'accentue d'année en année (Ouédraogo et al.., 2006). D’autres causes notamment anthropiques, ont déjà été largement évoquées par de nombreux auteurs pour expliquer la dégradation du peuplement ligneux. Il s'agit entre autres, de la forte sollicitation des espèces ligneuses locales dans plusieurs domaines d'usages (Traore, 2008) entraînant ainsi leur surexploitation (Thiombiano et al., 2012). Les espèces ligneuses indigènes jouent un rôle important en complément du régime alimentaire des populations rurales au Niger, en particulier en période de pénurie alimentaire Agúndez et al. (2016). D’autres études (Lykke, 2000; Tente et al., 2002 ; Douma et al., 2010) ont montré que les activités humaines ont un impact négatif sur la structure, la composition floristique et la dynamique des écosystèmes forestiers. L'impact le plus visible est la régression de certaines espèces ligneuses corrélée à l'expansion d'autres (Akpo et al., 1996).

Le problème fondamental qui se pose est le prélèvement sélectif de certaines espèces prioritaires pour les populations locales qui menace dangereusement les ressources phytogénétiques locales. En effet, comme l'affirment Ado et al. (2016), la surexploitation des espèces de soudure combinée au changement climatique ne semble pas garantir l'utilisation durable de ces essences forestières. A titre indicatif, selon Douma (2016), les espèces les plus menacées, sont entre autres Parkia biglobosa, Vittelaria paradoxa, Tamarindus indica, Adansonia digitata, dans la zone Nord soudanienne et Maerua crassifolia, Boscia senegalensis, et Balanites aegytiaca en zone sahélienne.

Le Dallo Bosso, communément appelé Boboye, est une vallée fossile située dans les régions de Tillabéri et Dosso. Son peuplement ligneux est en proie à une forte exploitation par une population à la recherche de compléments alimentaires et des sources de revenus et à une forte pression pastorale (Dan Guimbo et al., 2012).

L'espèce Neocarya macrophylla ou pommier du Cayor, arbre et arbuste de la famille des Chrysobalanacées est la plus touchée. Elle compte parmi les espèces ligneuses les plus caractéristiques du Dallol Bosso. C'est une espèce oléagineuse qui forme avec les cultures annuelles, un type physionomique de parc agroforestier (Dan Guimbo et al., 2010) et fait partie des ressources les plus appréciées dans la zone (Balla et al., 2008).

La connaissance ethnobotanique de l'espèce permettra de mieux appréhender le niveau de menace et de proposer des stratégies de sa gestion durable. D'où tout l'intérêt du présent travail qui a pour but d'analyser l'état actuel des populations de Neocarya macrophylla dans les terroirs du Dallol Bosso afin de connaitre la perception locale du niveau de menace sur l'espèce. 


\section{Milieu d'étude}

L'étude s'est déroulée dans les deux zones climatiques (soudanienne et sahélienne) que parcourt le Dallol Bosso.

Avec une orientation Nord-Sud, cette vallée fossile s'étend sur une longueur de $330 \mathrm{~km}$ de la frontière du Mali jusqu'au fleuve Niger à la frontière du Bénin. Elle concentre la majorité de la population des départements de Filingué et de Boboye.

Le Dallol Bosso (Figure 1) constitue la partie inférieure du cours de la rivière fossile Azawak.

Le mot « Dallol » signifie « vallée » dans la langue Peulh, tandis que «Boboye » est le terme équivalent dans la langue Zarma.

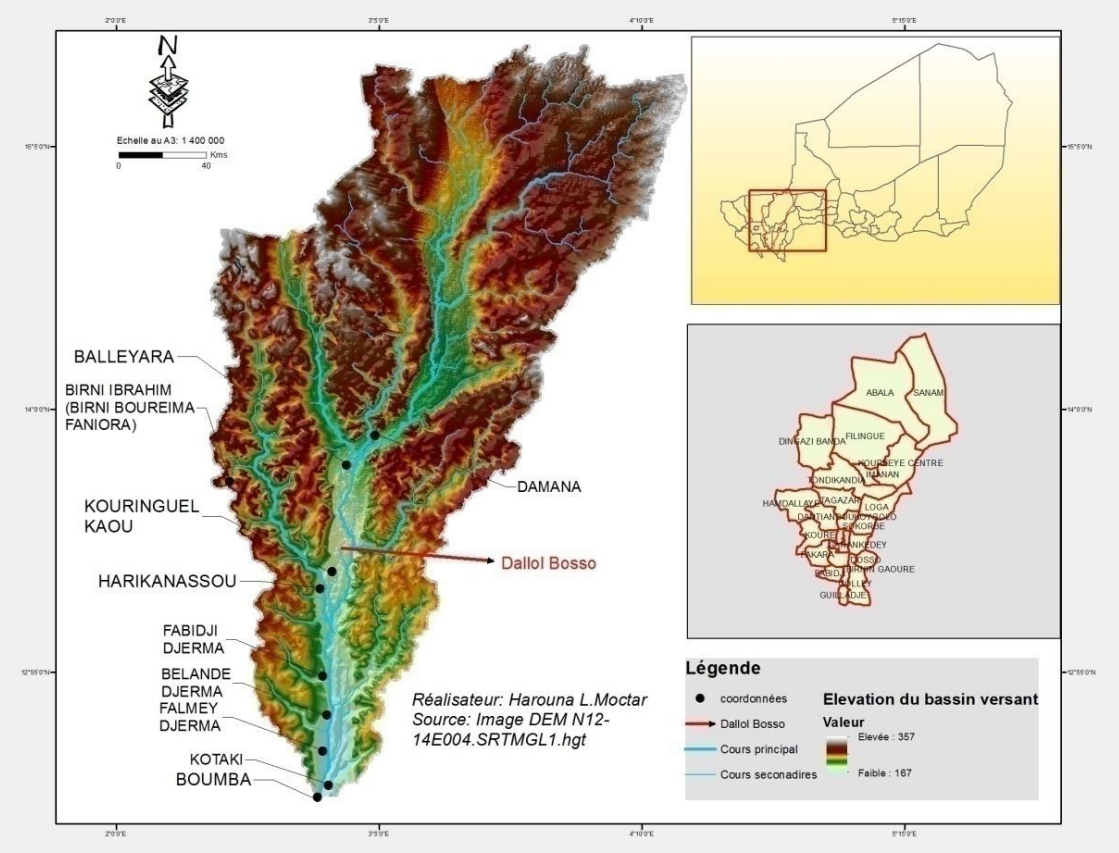

Figure 1 : Carte de la zone d'étude

\section{Méthodes}

La méthode utilisée a consisté à faire des enquêtes en milieu naturel dans les localités réparties dans toute la vallée du Dallol Bosso. Dix villages ont été choisis le long du Dallol Bosso, ce sont : Boumba, Kotaki, Falmeye, Bélandé, Fabirdji, Birni N'Gaouré, Harikanassou, Kouringuel, Balayara et Damana (Carte 1). Le choix de ces localités a tenu compte de l'homogénéité du peuplement ligneux des deux zones climatiques. Au niveau des localités, des enquêtes socio-économiques ont été réalisées auprès des populations, à l'aide d'un questionnaire. Le choix des personnes enquêtées est basé sur deux (2) critères essentiels : (1) l'unité d'enquête qui est le chef de ménage ; le 
ménage étant défini ici comme un groupe personnes qui vivent sous le même toit, (2) la disponibilité et le consentement des personnes enquêtées.

L'échantillon a été constitué avec cent (100) chefs de ménages à travers les dix (10) villages. Dans chaque village nous avons enquêté dix chefs de ménage.

Ainsi, les informations collectées ont porté sur les espèces ligneuses alimentaires, leur état, les organes prélevés, les préférences, les facteurs de menaces, etc. En ce qui concerne l'appréciation des plantes, une classification préférentielle a été réalisée pour établir un ordre de préférence entre les plantes. Cette classification a consisté à attribuer des scores aux différentes plantes (Belem et al., 2008). A chaque plante, il a été attribué par répondant un score allant de 1 à 10. L'espèce la plus importante a été classée au premier rang (1) ainsi de suite jusqu'à la moins importante qui a reçu un rang de 10 . Pour ce qui est de l'appréciation de la menace, elle a consisté à donner des points de 0 à 4 pour tous les ligneux alimentaires de soudure pour désigner le niveau de menace. Le point $0=$ plante pas menacée, $1=$ menace pas importante, 2 = quelque peu menacée ; $3=$ assez menacée, $4=$ aucune réponse.

Par ailleurs, les observations ont été effectuées sur les arbres pour identifier les formes de menaces et les prendre en image.

Les données recueillies ont été codifiées, puis saisies pour aboutir à une base de données sous format Excel. La statistique descriptive a été effectuée à l'aide du logiciel Excel 2007.

\section{Résultats}

\section{Importance de l'espèce dans le peuplement ligneux}

Au total, vingt-quatre (24) espèces ligneuses réparties en vingt-un (21) genres et treize (13) familles sont consommées par les populations locales de la zone d'étude. Il y a dix-huit (18) espèces communes aux deux zones. La consommation varie suivant les zones climatiques. Ainsi les espèces les plus citées au niveau de la zone soudanienne sont: Neocarya macrophylla $(17,69 \%)$, Vittelaria paradoxa $(14,60 \%)$ et Borassus aethiopum $(10,61 \%)$.

En zone sahélienne: Balanites aegyptiaca (20,14\%), Neocarya macrophylla (16,41\%), Hyphaene thebeica $(14,17 \%)$ sont les espèces dominantes (tableau I).

On constate qu'il y a plus d'espèces en zone soudanienne qu'en zone sahélienne. Aussi, en zone soudanienne, Neocarya macrophylla reste l'espèce dominante tandis qu'elle occupe le deuxième rang dans la zone sahélienne. 
Tableau I : Espèces ligneuses alimentaires citées par les populations de la zone d'étude.

\begin{tabular}{|c|c|c|c|c|c|}
\hline \multirow[t]{2}{*}{ Nom scientifique } & \multirow[t]{2}{*}{ Nom de famille } & \multicolumn{2}{|c|}{ Zone soudanienne } & \multicolumn{2}{|c|}{ Zone sahélienne } \\
\hline & & $\begin{array}{l}\text { Nombre } \\
\text { de } \\
\text { citation }\end{array}$ & $\begin{array}{l}\text { Fréquence } \\
(\%)\end{array}$ & $\begin{array}{l}\text { Nombre } \\
\text { de } \\
\text { citation }\end{array}$ & $\begin{array}{l}\text { Fréquence } \\
\quad(\%)\end{array}$ \\
\hline $\begin{array}{l}\text { Neocarya macrophylla } \\
\text { (Sabine) Prance }\end{array}$ & Chrysobalanaceae & 40 & 17,69 & 22 & 16,41 \\
\hline Borassus aethiopum Mart & \multirow{3}{*}{ Arecaceae } & 24 & 10,61 & 3 & 2,22 \\
\hline Phoenix dactylifera $L$. & & 1 & 0,44 & 1 & 0,74 \\
\hline Hyphaene thebeica(L.)Mart & & 22 & 9,73 & 19 & 14,17 \\
\hline Vitellaria paradoxa C.F.Gaertn & Sapotaceae & 33 & 14,60 & 1 & 0,74 \\
\hline Vitex doniana Sweet & Verbenaceae & 17 & 7,52 & 7 & 5,22 \\
\hline Ziziphus mauritiana Lam. & Rhamnaceae & 14 & 6,19 & 18 & 13,43 \\
\hline $\begin{array}{c}\text { Sarcocephalus latifolius (Sm.) } \\
\text { E.A.Bruce }\end{array}$ & Rhubiaceae & 1 & 0,44 & 1 & 0,74 \\
\hline $\begin{array}{c}\text { Diospyros mespiliformis } \\
\text { Hochst.ExA. DC }\end{array}$ & Ebenaceae & 14 & 6,19 & 2 & 1,49 \\
\hline Ceiba pentandra (L.) Gaertn & \multirow[t]{2}{*}{ Bombacaceae } & 1 & 0,44 & 0 & 0 \\
\hline Adansonia digitata $L$. & & 3 & 1,32 & 3 & 2,23 \\
\hline $\begin{array}{c}\text { Balanites aegyptiaca }(\mathrm{L} .) \\
\text { Delile }\end{array}$ & Balanitaceae & 22 & 9,73 & 27 & 20,14 \\
\hline $\begin{array}{c}\text { Daniellia oliveri (Rolfe) Hutch. } \\
\text { \& Dalziel }\end{array}$ & \multirow{4}{*}{$\begin{array}{l}\text { Fabaceae- } \\
\text { Caesalpinoideae }\end{array}$} & 7 & 3,09 & 0 & 0 \\
\hline $\begin{array}{c}\text { Detarium microcarpum Guill. } \\
\text { \&Perr }\end{array}$ & & 7 & 3,09 & 5 & 3,73 \\
\hline Tamarindus indica $L$. & & 6 & 2,65 & 2 & 1,49 \\
\hline $\begin{array}{c}\text { Piliostigma reticulatum (DC.) } \\
\text { Hochst }\end{array}$ & & 6 & 2,65 & 2 & 1,49 \\
\hline Anacardium occidentale $L$. & \multirow{3}{*}{ Anacardiaceae } & 1 & 0,44 & 0 & 0 \\
\hline $\begin{array}{c}\text { Sclerocarya birrea (A.Rich.) } \\
\text { Hochst }\end{array}$ & & 2 & 0,88 & 6 & 4,47 \\
\hline $\begin{array}{c}\text { Lannea microcarpa Engl. \& K. } \\
\text { Krause }\end{array}$ & & 1 & 0,44 & 2 & 1,49 \\
\hline Annona senegalensis Pers. & Annonaceae & 1 & 0,44 & 4 & 2,98 \\
\hline Ficus tonninjii & \multirow{4}{*}{ Moraceae } & 1 & 0,44 & 0 & 0 \\
\hline Ficus ingens (Miq.) Miq. & & 1 & 0,44 & 0 & 0 \\
\hline Ficus platyphylla Delile & & 1 & 0,39 & 5 & 3,73 \\
\hline $\begin{array}{c}\text { Ficus gnaphalocarpa (Miq.) } \\
\text { Miq }\end{array}$ & & 0 & 0 & 4 & 2,98 \\
\hline $\begin{array}{l}\text { Total } \\
\end{array}$ & & 226 & 100 & 134 & 100 \\
\hline
\end{tabular}

\section{Provenance des organes des espèces prioritaires}

La figure 2 montre les principales sources d'approvisionnement des organes consommés.

La brousse et les marchés constituent les principales provenances des organes utilisés dans l'alimentation humaine avec respectivement des 
proportions de $28,32 \%$ et $28,13 \%$. Ils sont suivis des champs $(19,27 \%)$.

L'espèce Neocarya macrophylla est une plante recherchée surtout pour ses fruits. A l'instar des autres espèces ligneuses, selon les ménages enquêtés, ses produits proviennent en grande partie de la brousse $(33,33 \%)$ suivies des marchés $(27,45 \%)$ où ils sont vendus principalement par les femmes et les enfants et des champs $(25 \%)$.

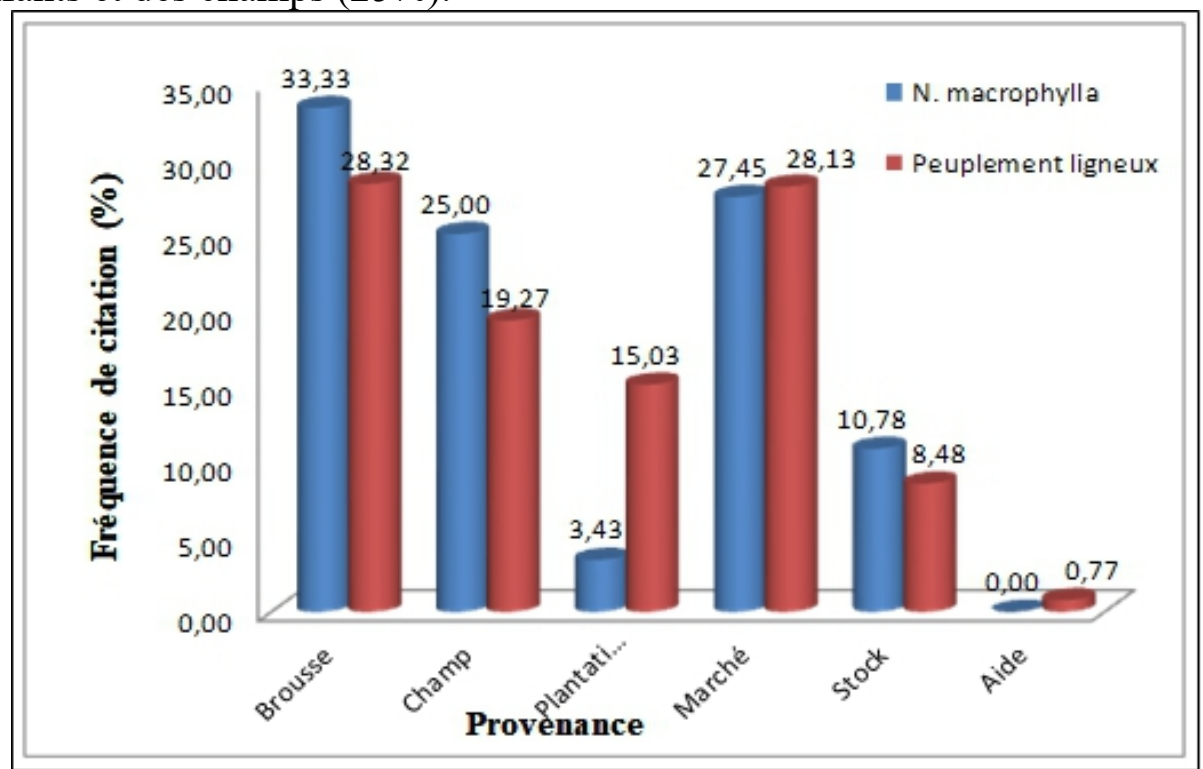

Figure 2 : Provenance des organes des espèces prioritaires et de Neocarya macrophylla

\section{Principaux organes prélevés sur les espèces}

Le peuplement ligneux de la vallée fournit des produits et services aux populations locales. Les principales parties couramment utilisées pour des fins alimentaires et médicinales sont : les feuilles, les fleurs, les fruits, les écorces et les racines. Les fruits sont les plus exploités avec une fréquence de 55,86\% suivis de l'écorce et des feuilles avec respectivement des proportions de $22,85 \%$ et $15,24 \%$. Les fleurs sont les organes les moins utilisés selon les populations enquêtées.

En ce qui concerne l'espèce Neocarya macrophylla il ressort que les fruits sont exploités à 82,56\%. L'utilisation de l'écorce occupe la deuxième place $(10,47 \%)$ suivie de celle des feuilles qui est à 3,49\%. Les fleurs sont les moins exploités avec des proportions de réponses de 1,16\% (figure 3 ). 


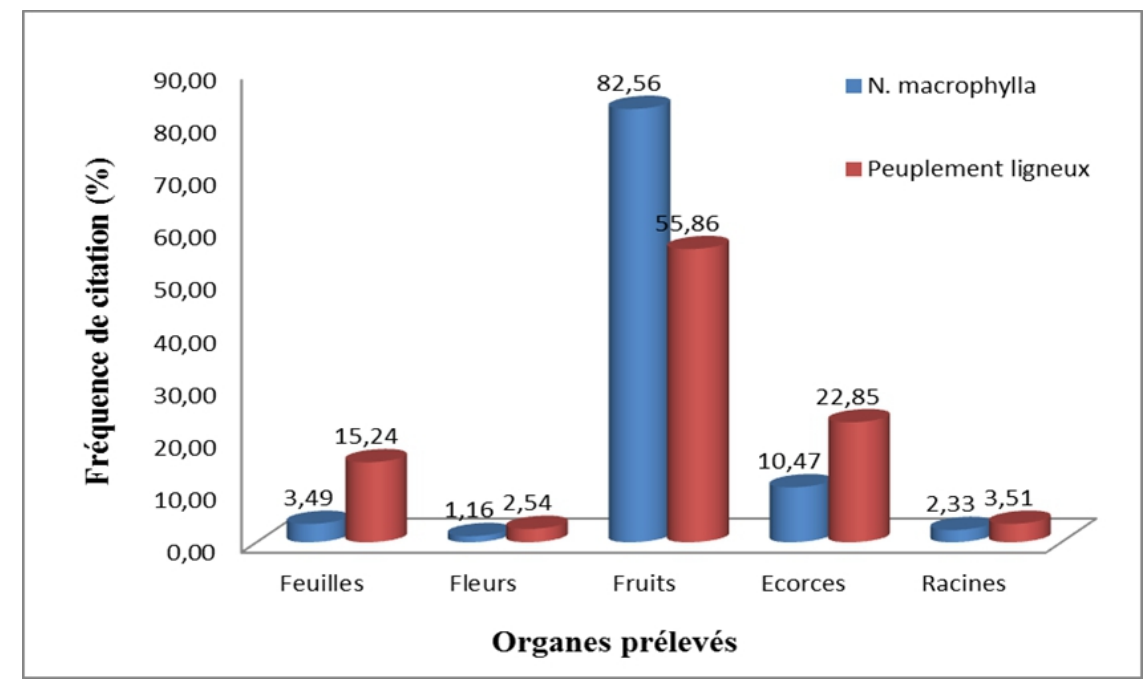

Figure 3 : Principaux organes prélevés sur les espèces prioritaires et sur Neocarya macrophylla.

La photo 1 montre des pieds de Neocarya macrophylla dans un champ formant un système agroforestier. La photo 2 ci-dessous est celle des fruits mûrs du pommier de Cayor (Neocarya macrophylla).
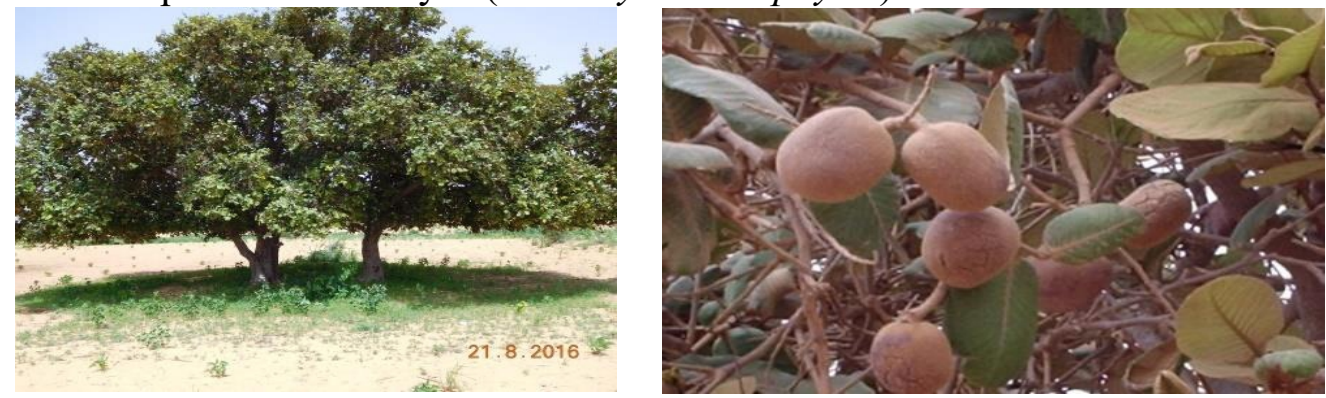

Photo1 : Pieds de N. macrophylla dans un champ. Photo2 : Fruits de N. macrophylla.

\section{Niveau de priorité de l'espèce dans l'alimentation}

Les espèces les plus appréciées dans la zone soudanienne sont Neocarya macrophylla, Vittelaria paradoxa, Borassus aethiopum et Hyphaene thebeica avec respectivement les scores de 40, 33, 24 et 22.

En zone sahélienne, les espèces les plus prisées sont Balanites aegyptiaca, Neocarya macrophylla, Hyphaene thebeica, Ziziphus mauritiana avec des scores respectifs de 27, 22, 19 et 18 (tableau II).

Tableau II: Espèces ligneuses alimentaires prioritaires

\begin{tabular}{|c|c|c|c|c|c|c|}
\hline Code & Noms botaniques & $\begin{array}{c}\text { Familles } \\
\text { botaniques }\end{array}$ & $\begin{array}{c}\text { Noms } \\
\text { locaux } \\
\text { (Zarma) }\end{array}$ & Organes consommés & Score & Rang \\
\hline \multicolumn{6}{|c|}{ Zone Soudanienne } \\
\hline Nema & Neocarya macrophylla & Chrysobalanaceae & $\begin{array}{c}\text { Gamsa- } \\
\text { gna }\end{array}$ & Fruits & 40 & 1 \\
\hline
\end{tabular}




\begin{tabular}{|c|c|c|c|c|c|c|}
\hline Vipa & Vittelaria paradoxa & Verbenaceae & $\begin{array}{l}\text { Boulanga- } \\
\text { gna }\end{array}$ & Fruits & 33 & 2 \\
\hline Boae & Borassus aethiopum & Arecaceae & $\begin{array}{l}\text { Sabizé- } \\
\text { gna }\end{array}$ & Fruits & 24 & 3 \\
\hline Hyth & Hyphaene thebeica & Palmae & $\begin{array}{c}\text { Kangaou- } \\
\text { gna }\end{array}$ & Fruits & 22 & 4 \\
\hline Baae & Balanites aegyptiaca & Balanitaceae & $\begin{array}{c}\text { Garbey- } \\
\text { gna }\end{array}$ & Fruits & 22 & 5 \\
\hline Vido & Vitex doniana & Verbenaceae & Boyi-gna & Fruits & 17 & 6 \\
\hline Zima & Ziziphus mauritiana & Rhamnaceae & $\begin{array}{c}\text { Darey- } \\
\text { gna }\end{array}$ & Fruits & 14 & 7 \\
\hline Dime & Diospyros mespiliformis & Ebenaceae & $\begin{array}{c}\text { Tokey- } \\
\text { gna }\end{array}$ & Fruits & 14 & 8 \\
\hline Daol & Daniellia oliveri & Caesalpiniaceae & $\begin{array}{c}\text { Falmey- } \\
\text { gna }\end{array}$ & Fruits & 7 & 9 \\
\hline Demi & Detarium microcarpum & Caesalpiniaceae & $\begin{array}{l}\text { Fantou- } \\
\text { gna }\end{array}$ & Fruits & 7 & 10 \\
\hline \multicolumn{7}{|c|}{ Zone Sahélienne } \\
\hline Baae & Balanites aegyptiaca & Balanitaceae & $\begin{array}{l}\text { Garbey- } \\
\text { gna }\end{array}$ & Fruits & 27 & 1 \\
\hline Nema & Neocarya macrophylla & Chrysobalanaceae & $\begin{array}{c}\text { Gamsa- } \\
\text { gna }\end{array}$ & Fruits & 22 & 2 \\
\hline Hyth & Hyphaene thebeica & Palmae & $\begin{array}{c}\text { Kangaou- } \\
\text { gna }\end{array}$ & Fruits & 19 & 3 \\
\hline Zima & Ziziphus mauritiana & Rhamnaceae & $\begin{array}{c}\text { Darey- } \\
\text { gna }\end{array}$ & Fruits & 18 & 4 \\
\hline Vido & Vitex doniana & Verbenaceae & Boyi-gna & Fruits & 7 & 5 \\
\hline Scbi & Sclerocarya birrea & Anacardiaceae & $\begin{array}{c}\text { Diney- } \\
\text { gna }\end{array}$ & Fruits & 6 & 6 \\
\hline Demi & Detarium microcarpum & Caesalpiniaceae & $\begin{array}{c}\text { Fantou- } \\
\text { gna }\end{array}$ & Fruits & 5 & 7 \\
\hline Fipl & Ficus platyphylla & Moraceae & $\begin{array}{c}\text { Kobbé- } \\
\text { gna }\end{array}$ & Fruits & 5 & 8 \\
\hline Fign & Ficus gnaphalocarpa & Moraceae & $\begin{array}{c}\text { Gaigai- } \\
\text { gna }\end{array}$ & Fruits & 4 & 9 \\
\hline Anse & Annona senegalensis & Annonaceae & Mufa-gna & Fruits & 4 & 10 \\
\hline
\end{tabular}

\section{Importance alimentaire et menaces sur les espèces ligneuses}

Les investigations ont permis de recenser onze (11) espèces prioritaires qui semblent être menacées dans les zones soudanienne et sahélienne. Parmi ces espèces, Neocarya macrophylla, espèce présente dans les deux zones est menacée par plusieurs facteurs dont : le défrichage pour la mise en culture des terres, l'exploitation du bois, la sécheresse et l'appauvrissement des sols (tableau III). 
Tableau III : Degré des menaces des espèces ligneuses dans les zones soudanienne et sahélienne

\begin{tabular}{|c|c|c|c|c|c|c|c|c|c|c|}
\hline Espèces & Zone & Déf & Pat & $\begin{array}{l}\text { Exp } \\
\text { Bois }\end{array}$ & $\begin{array}{l}\text { Exp } \\
\text { Fr, Fl }\end{array}$ & $\begin{array}{l}\text { Exp } \\
\text { Eco }\end{array}$ & $\begin{array}{l}\text { Prod } \\
\text { Char }\end{array}$ & $\begin{array}{l}\text { Inse et } \\
\text { Mala }\end{array}$ & Séch & $\begin{array}{l}\text { Sol } \\
\text { appv } \\
\text { et } \\
\text { Engr }\end{array}$ \\
\hline N. macro & $\mathrm{Sh} / \mathrm{Sd}$ & 3 & 2 & 3 & 3 & 1 & 2 & 1 & 2 & 1 \\
\hline V.paradox & $\mathrm{Sh} / \mathrm{Sd}$ & 0 & 3 & 0 & 3 & 2 & 0 & 2 & 3 & 1 \\
\hline B.aethiop & $\mathrm{Sh} / \mathrm{Sd}$ & 0 & 0 & 2 & 3 & 2 & 0 & 0 & 3 & 0 \\
\hline H.thebeia & $\mathrm{Sh} / \mathrm{Sd}$ & 0 & 0 & 1 & 0 & 1 & 0 & 0 & 2 & 1 \\
\hline B.aegyp & $\mathrm{Sh} / \mathrm{Sd}$ & 0 & 0 & 0 & 3 & 2 & 0 & 1 & 2 & 0 \\
\hline V.doniana & $\mathrm{Sh} / \mathrm{Sd}$ & 3 & 0 & 2 & 0 & 1 & 0 & 3 & 2 & 0 \\
\hline Z.mauritia & $\mathrm{Sh} / \mathrm{Sd}$ & 3 & 0 & 0 & 0 & 0 & 0 & 3 & 2 & 2 \\
\hline D.mespili & $\mathrm{Sd} / \mathrm{Sh}$ & 1 & 0 & 1 & 0 & 0 & 0 & 0 & 2 & 0 \\
\hline S.birrea & $\mathrm{Sh} / \mathrm{Sd}$ & 0 & 0 & 3 & 0 & 0 & 3 & 0 & 2 & 0 \\
\hline D.microca & $\mathrm{Sh} / \mathrm{Sd}$ & 0 & 0 & 3 & 0 & 0 & 3 & 0 & 0 & 0 \\
\hline F.platyph & $\mathrm{Sh} / \mathrm{Sd}$ & 0 & 0 & 2 & 0 & 1 & 2 & 0 & 2 & 1 \\
\hline
\end{tabular}

Sd: soudanienne; Sh: sahélienne; $\mathrm{Sd} / \mathrm{Sh}$ : soudanienne et sahélienne; $0=$ pas menacée; 1 = pas important; 2 = quelque peu menacée; $3=$ menace très importante; $4=$ aucune idée; Déf $=$ défrichage $;$ Pat $=$ pâturage $;$ Exp bois $=$ exploitation du bois; Exp fr, $\mathrm{fl}=$ exploitation des fruits et fleurs; Exp eco = exploitation des écorces; Prod char = production de charbon; Inse et mala $=$ attaques des insectes et des maladies; Séch $=$ sécheresse; Sol appv et engr $=$ sol appauvri et influence des engrais.

Ainsi, Vittelaria paradoxa et Borassus aethiopum sont menacées par trois facteurs : l'exploitation des fruits et des fleurs, celle de l'écorce et la sécheresse.

Hyphaene thebeica est menacée par l'exploitation du bois, le prélèvement de l'écorce, de l'appauvrissement des sols et la sécheresse.

Balanites aegyptiaca est extrêmement menacée avec un degré (3) par l'exploitation des fruits et fleurs ; il est souvent aussi victime d'écorçage. La sécheresse a été aussi citée comme facteur de menace comme pour l'ensemble des espèces.

Les espèces Vitex doniana et Ziziphus mauritiana sont tributaires du défrichage, de l'attaque des insectes et maladies, de la sécheresse et de l'appauvrissement.

Le défrichage, l'exploitation du bois et la sécheresse sont les potentielles menaces pour Diospyros mespiliformis.

L'exploitation du bois et la production du charbon font que Sclerocarya birrea et Detarium microcarpum sont considérablement menacées (degré 3).

A travers l'analyse de ce tableau, Ficus platyphylla est menacée par l'exploitation du bois (degré 2), l'exploitation de l'écorce (degré 1), la production du charbon et la sécheresse avec degré de menaces (2). Il est enfin 
menacé par l'appauvrissement des sols même si cette forme de menace n'a pas beaucoup d'impacts (degré 1).

Toutes ces dernières espèces se retrouvent dans les deux zones agroécologiques (soudanienne et sahélienne).

\section{Constat des menaces sur le terrain}

Le constat des menaces révèle que la production artisanale du natron constitue la plus grande menace sur Neocarya macrophylla dans le Dallol Bosso. En effet, les femmes qui en sont les productrices s'approvisionnent en bois de chauffe en coupant abusivement les racines des pieds vivants qu'elles font sécher ensuite pour faire cuire les eaux natronées (photo 3).

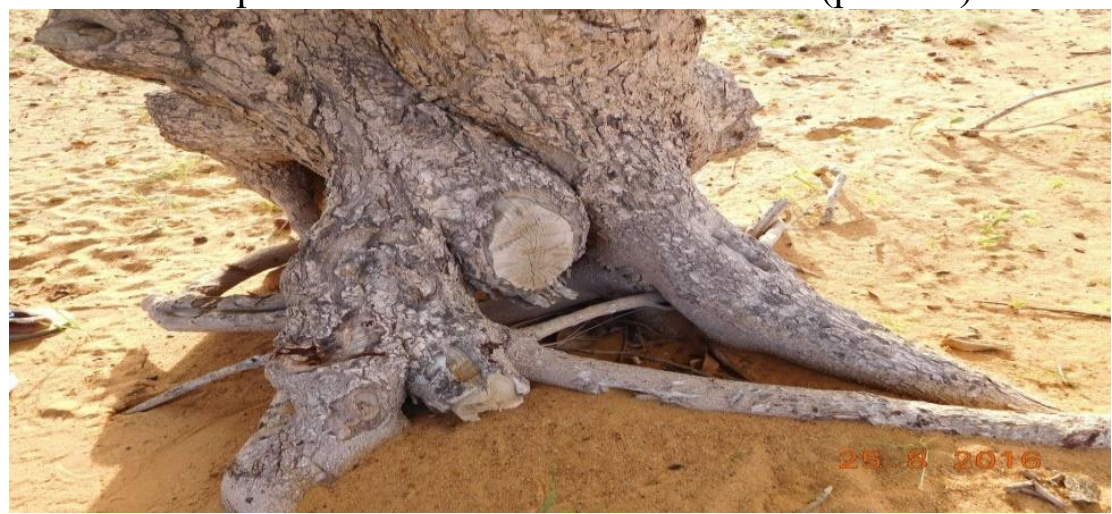

Photo 3 : Coupe abusive des racines du N. macrophylla

Les érosions contribuent au déracinement des pieds (photo 4), facilitant souvent leur chute (photo 5). La population du Neocarya macrophylla est aussi menacée de disparition du fait du lotissement des terrains.

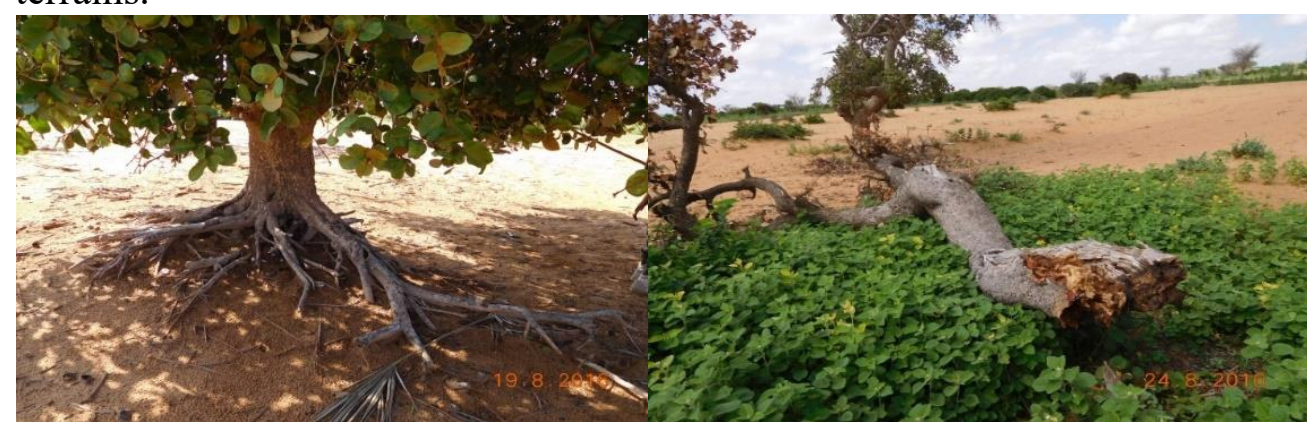

Photo 4 : Effet de l'érosion sur N. macrophylla Photo 5 : Chute d'un pied de $N$. macrophylla

Dans les champs, la partie aérienne des pieds répartis en agrégat est souvent supprimée par les paysans pour réduire l'effet de compétition de lumière avec les cultures ou pour stocker les résidus des récoltes comme les fans de niébé et de mil. Les tiges servent aussi de clôture pour les maisons et 
les greniers.

L’écorce utilisée dans la pharmacopée traditionnelle est prélevée abusivement (photo 6).

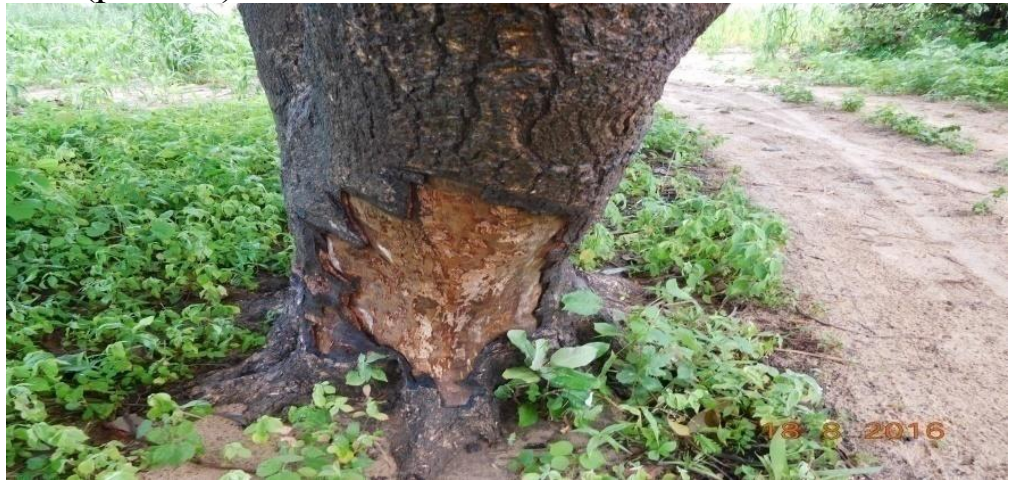

Photo 6 : Ecorçage du N. macrophylla

\section{Discussion}

La présente étude nous renseigne sur la diversité des espèces ligneuses alimentaires et des utilisations au niveau local mais aussi sur la perception locale du niveau de menace. Au total, il a été recensé 24 espèces ligneuses dans l'ensemble des écosystèmes de la vallée du Dallol Bosso. Ainsi, vingttrois (23) espèces ont été répertoriées en zone soudanienne contre dix-neuf (19) au niveau de la zone sahélienne et dix-huit (18) sont communes aux deux zones agro écologiques. Les espèces de cette liste figurent déjà sur la liste nationale des espèces alimentaires. Selon Douma (2016), de manière générale les populations reconnaissent 26 espèces en zone soudanienne, 17 espèces en zones sahélienne et 11 espèces communes aux deux zones agro-écologiques comme espèces alimentaires de soudure. Plusieurs travaux ont montré l'importance du commerce et/ou la consommation des produits de ces arbres pour les ménages (Douma, 2016). Leur disponibilité est sous la dépendance des saisons. L'étude a mis aussi en évidence la variation de la diversité des espèces entre les deux zones. Ainsi, il y a plus d'espèces alimentaires en zone soudanienne qu'en zone sahélienne. Cette différence peut s'expliquer par le fait qu'en zone soudanienne, les précipitations sont plus élevées qu'au niveau de la zone sahélienne. Le caractère superficiel de la nappe phréatique dans la zone soudanienne fait aussi de cette entité agro-écologique un milieu favorable à la croissance et au développement de certaines espèces ligneuses notamment Neocarya macrophylla. Ces résultats corroborent ceux trouvés par Abdou (2009) qui rapporte que les populations de la zone du Boboye ont avancé que la faible profondeur de la nappe phréatique de la vallée du Dallol constitue un facteur d'installation d'une population dense de $N$. macrophylla. Les sources de prélèvement des organes varient en fonction de la disponibilité des espèces dans les milieux. En effet, l'étude a révélé que la brousse et les marchés 
constituent les principales provenances des organes utilisés dans l'alimentation humaine avec respectivement $28,32 \%$ et $28,13 \%$. Cette situation peut se justifier par l'abondance des peuplements naturels d'arbres au niveau des brousses moins antrhopisées d'une part et d'autre part le revenu substantiel qu'offre la vente des fruits et des amandes de l'espèce sur les marchés locaux et urbains. Cette prédominance de Neocarya macrophylla corrobore les résultats d'Abdou (2009) dans la même zone. En effet, cet auteur rapporte que le peuplement ligneux dans les espaces de pâture est caractérisé par une espèce dominante, Neocarya macrophylla avec une contribution spécifique de $59,13 \%$.

L'utilisation des produits de l'espèce est fonction de la disponibilité et des besoins immédiats des populations. Ainsi, les fruits qui sont disponibles presque toute l'année restent l'organe le plus prisé $(55,86 \%)$ suivi des écorces $(22,85 \%)$ et des feuilles $(15,24 \%)$. Cela se justifie d'autant plus que les fruits et les amandes constituent des compléments alimentaires en période de soudure. Le fruit a un goût sucré et aromatisé surtout en période sèche et vers les mois de décembre à février (Balla et al., 2008). Quant aux feuilles et écorces, elles sont appréciées pour leurs vertus médicinales (Thiombiano et al., 2010). Les espèces alimentaires citées comme prioritaires par les populations au niveau de la zone soudanienne sont entre autres : Neocarya macrophylla (17,69\%),Vitellaria paradoxa (14,60\%), Borassus aethiopum (10,61\%) et Hyphaene theibeca (9,73\%). C'est surtout la consommation des fruits qui fait de ces espèces prioritaires. En ce qui concerne la zone sahélienne, ce sont: Balanites aegyptiaca (20,14\%), Neocarya macrophylla $(16,41 \%)$, Hyphaene thebeica $(14,17 \%)$ et Ziziphus mauritiana $(13,43 \%)$. Cette variation entre les deux zones serait due aux exigences écologiques qui déterminent pour chaque espèce une aire de de répartition bien précise.

«Le Gamsa est plus qu'un arbre chez nous, mais une source de vie», affirme un paysan. Cela indique que les populations locales tirent profit de cette espèce ligneuse. C'est dans le même ordre d'idée que Seyni (2009) a rapporté que dans le Sud-ouest du Niger, N. macrophylla se présente comme une espèce à usages multiples. La menace potentielle selon les populations de la zone soudanienne est l'exploitation du bois, des fruits et des fleurs, suivie du défrichage et de l'exploitation des feuilles. Par contre, dans la zone sahélienne, les sécheresses, l'exploitation des fruits et des fleurs, les attaques des maladies et des insectes constituent les véritables menaces pour les espèces ligneuses. Les sécheresses récurrentes constituent l'origine de ces menaces car celles-ci entrainent une baisse considérable des rendements des cultures, contraignant ainsi les populations locales à s'attaquer aux produits issus des espèces ligneuses. Ces résultats confirment ceux trouvés par Douma (2016) qui indique que ces facteurs sont tous liés, quelle que soit la zone 
écologique, aux sécheresses qui génèrent des baisses considérables de rendement. Cela oblige les populations aux cueillettes des parties comestibles des plantes comme les feuilles, fleurs et fruits, l'exploitation répétée de cellesci entraine des attaques d'insectes nuisibles pouvant conduire au dépérissement des arbres. Particulièrement en zone soudanienne où elles sont prédominantes, les espèces comme $N$. macrophylla, $B$. aethiopum, $B$. aegyptiaca et $Z$. mauritiana sont menacées à des degrés 2 et 3 . Les causes de ces menaces sont l'exploitation abusive du bois pour les constructions, le défrichage, la sécheresse ainsi que l'appauvrissement des sols au niveau de la première espèce. En effet, Dan Guimbo et al. (2017) affirment que le pommier de Cayor est soumis à diverses pressions humaines telles que l'écorçage et la coupe pour la fabrication des mortiers qui se fait avec du bois frais, facile à manipuler.

B. aethiopum est menacée par la forte exploitation du bois, des fruits et fleurs, des écorces et de la sécheresse. B. aegyptiaca est menacée par l'exploitation abusive de ses fleurs et fruits pour l'alimentation humaine. Quant au Z. mauritiana, ce sont les maladies et les insectes qui constituent le facteur de menace; selon Dan Guimbo et al. (2012), la pollinisation insuffisante, la limitation des ressources nutritives, les prédations des organes de reproduction et les conditions climatiques défavorables sont les facteurs qui expliquent les défauts de fructification de certaines espèces d'arbres pendant plusieurs saisons. D'après Laouali et al. (2014) chez Prosopis africana, certaines formes d'exploitation, associées aux problèmes naturels de régénération et de germination contribuent à rendre plus graves les menaces qui pèsent sur la survie de cette espèce.

\section{Conclusion}

L'enquête ethnobotanique menée dans la zone du Dallol Bosso montre une diversité d'espèces consommées par la population. Ces ligneux de grande importance dans la vie socio-économique des populations, font l'objet de plusieurs usages. Les produits forestiers non ligneux comestibles contribuent à l'amélioration de la sécurité et la diversité des régimes alimentaires, l'équilibre nutritionnel et l'amélioration des revenus monétaires des ménages. Par contre, la surexploitation de ces ligneux et les conditions climatiques défavorables conduisent à leur détérioration. Il est donc nécessaire de mener des recherches approfondies pour que ces produits puissent être utilisés bénéfiquement sans pour autant menacer l'avenir du peuplement.

\section{References:}

1. Abdou D., 2009. Caractérisation des parcs agroforestiers à Neocarya macrophylla dans le Boboye, usages et formes d'utilisation : cas des terroirs de Kouringuel, Gamsazougou et Balla koira. Mémoire d'ITA, 
Faculté d'Agronomie, UAM, 46 pages.

2. Agúndez D., Douma S., Madrigal J., Gómez-Ramos A., Vinceti B., Ricardo A. and Mahamane A., 2016. Conservation of food tree species in Niger: towards a participatory approach in rural communities. Forest Systems. Volume 25 • Issue 3 • e080 25(3), 13 pages eISSN: 2171-9845.

3. Akpo L. E., Bada F. et Grouzis M., 2003. Diversité de la végétation herbacée sous arbre : variation selon l'espèce ligneuse en milieu sahélien. Candollea 58, pp 515-530.

4. Akpo L. E. et Grouzis M., 1996. Influence du couvert sur la régénération naturelle de quelques espèces ligneuses sahéliennes (Nord-Sénégal, Afrique occidentale) Webbia 50(2) : 247-263.

5. Ado A., Laouali A., Douma S., Mahamane A., Saadou M., 2016. Les ligneux alimentaires de soudure dans les communes rurales de Tamou et Tondikiwindi: diversité et structure des populations. Journal of Animal \&Plant Sciences. Vol.31, Issue 1: 4889-4900.

6. Balla A., Barage M., Larwanou M. et Adam T., 2008. Le savoir-faire endogène dans la valorisation alimentaire des fruits du pommier de Cayor (N. macrophylla) au Niger. Bulletin de la Recherche Agronomique du Bénin, 59 : 1-7.

7. Belem B., Smith-Olsen C., Theilade I., Bellefontaine R., Guinko S. et Lykke AM., 2008. Identification des arbres hors Forêts préférés des populations du Sanmatenga (Burkina Faso). Bois et Forêts des Tropiques $298: 53-60$.

8. Dan Guimbo I., Laouali A., Habou R., Mahamane A., Ambouta K.JM., 2017.Le pommier de Cayor, espèce emblématique du Dallol Bosso (Niger). Agronomie Africaine 29 (1) : 13 - 19.

9. Dan Guimbo I., Barage M. et Douma S., 2012. Etudes préliminaires sur l'utilisation alimentaire des plantes spontanées dans les zones périphériques du parc W du Niger. Int. J. Biol. Chem. Sci. 6(6): 40074017.

10. Dan Guimbo I., Mahamane A. et Ambouta K.J.M., 2010. Peuplement des parcs à Neocarya macrophylla (Sabine) Prance et à Vitellaria paradoxa (Gaertn. C.F.) dans le sud-ouest nigérien : diversité, structure et régénération. Int. J. Biol. Chem. Sci., 4 (5): 1706-1720.

11. Douma S., 2016. Etude ethnobotanique et écologique des espèces ligneuses alimentaires de soudure des systèmes agroforestiers du sudouest au Niger : diversité, importance et niveau de menace. Thèse de Doctorat, Faculté des Sciences et Techniques, UAM de Niamey, 86 pages.

12. Douma S., Chaibou R. Mahamane A., N'DA D., et Saadou M., 2010. Etat actuel de dégradation des populations de quatre Rev. Ivoir. Sci. 
Technol., $16: 191-210$.

13. Douma S., 2009. Ecologie de quatre espèces ligneuses dans la Réserve Totale de faune de Tamou: indicateurs de gestion durable des ressources forestières. Mémoire de DEA, Faculté des Sciences et Techniques, UAM de Niamey, 69 pages.

14. Habou R., Diouf A., Bationo B. A., Segla K. N., Adjonou K., Kokutse A. D., Radji R., Kokou K., Mahamane A., et Saadou M., 2015. Structure des peuplements naturels de Pterocarpus erinaceus Poir. dans le domaine soudanien, au Niger et au Burkina Faso. Bois et forêts des tropiques, 2015, $n^{\circ} 325$ (3).

15. Laouali A., Dan Guimbo I., Larwanou M., Inoussa M.M. \& Mahamane A., 2014. Utilisation de Prosopis africana (G. et Perr.) Taub. dans le sud du département d'Aguié au Niger : les différentes formes et leur importance. Int. J. Biol. Chem. Sci. 8 (3), 1065-1074.

16. Lykke A. M., 2000. Local perception of vegetation change and priorities for conservation of woody savanna vegetation in Senegal. Journal of Environmental Management, 59: 107-120.

17. Ouédraogo A., Thiombiano A., Hahn-Hadjali K. et Guinko S., 2006. Diagnostic de l'état de dégradation des peuplements de quatre espèces ligneuses en zone soudanienne du Burkina Faso. Sécheresse 17 (4) : $485-491$.

18. Seyni D., 2009. Etude des possibilités de propagation de Neocarya macrophylla (Sabine) Prance (1976) en zone sahélienne. Mémoire de l'obtention du Diplôme d'Ingénieur des Techniques Agricoles, option : Eaux et Forêts, Faculté d'Agronomie, UAM de Niamey, 35 pages.

19. Tente B. et Sinsin B., 2002. Diversité des structures des formations arborescentes du secteur de Perma-Toucountouna dans la chaîne de l'Atacora (Bénin). Etudes.flor.vég. Burkina Faso 6.

20. Traore L., 2008. Inventaire des espèces ligneuses utilitaires de la région Sud-Ouest du Burkina Faso et état des populations de trois espèces à haute valeur économique. Mémoire de DEA en Sciences Biologiques Appliquées. Université de Ouagadougou, Burkina Faso, 46p.

21. Thiombiano D.N.E., Dibong D.S., Boussim I.J., 2012. Le rôle des espèces ligneuses dans la gestion de la soudure alimentaire au Burkina Faso. Secheresse 23: 86-93.

22. Thiombiano D.N.E., Lamien N., Dibong S.D. et Boussim IJ., 2010. Etat des peuplements des espèces ligneuses de soudure des communes rurales de Pobé-Mengao et Nobéré (Burkina Faso). Journal of Animal and Plant Sciences 9 : 1104-11 